\title{
MANET, its types, Challenges, goals and Approaches: A Review
}

\author{
Kayna Mahajan ${ }^{1}$, Harjit Singh ${ }^{2}$ \\ ${ }^{1}$ Student, Guru Nanak Dev University, RC Gurdaspur \\ ${ }^{2}$ Assistant Professor, Guru Nanak Dev University, RC Gurdaspur
}

\begin{abstract}
MANET is a field of networking that deals with the mobiles nodes of communication.. In the MANET due to new advancement sensing capability has been embedded to the node so MANET can be deployed at any platform for performing various tasks. In the MANET networks due to mobility \& sensing power consumption is the major issue of communication b/w different nodes. In the process of MANET power consumed in sensing, transmission of data from one source to another. In this procedure various proactive \& reactive protocol are used that provide reliable communication. The major Concern of MANET is energy optimization. To overcome the energy consumption problem dynamic clustering or residual energy concept can be used that reduce the energy consumption in MANET.
\end{abstract}

Keywords: MANET, DSDV, CGSR, DSR, AODV

\section{Introduction}

\subsection{MANET}

A Mobile Ad-hoc Network (MANET) is a set of remote versatile hubs shaping an/element self-sufficient system. Hubs speak with one another without the mediation of concentrated access focuses or base stations. Because of the restricted transmission scope of remote system interfaces, numerous bounces are expected to trade information between hubs in the system. Portable Ad hoc Network is the quick becoming innovation from the previous 20 years. The addition in their notoriety is a result of the simplicity of arrangement, foundation less and their element nature. [1]

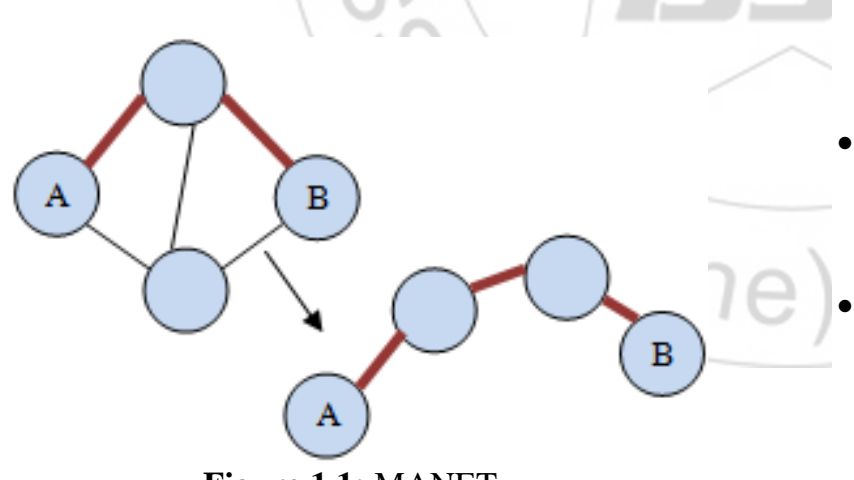

Figure 1.1: MANET

MANET's made another set of requests to be actualized and to give effective better end to end correspondence. The Dynamic Source Routing (DSR) Protocol is a source routed on-interest directing convention. A hub keeps up course reserves containing the source courses that it is mindful of. The hub overhauls entrances in the course reserve when it researches new courses. In its bundle head, every given directing parcel has a complete and requested hub list which the bundle will pass definitely.

\subsection{Types of MANET}

There are different types of MANETs including:
- In VANETs - Intelligent vehicular ad hoc networks make use of artificial intelligence to tackle unexpected situations like vehicle collision and accidents.

- Vehicular ad hoc networks (VANETs) - Enables effective communication with another vehicle or helps to communicate with roadside equipments.

- Internet Based Mobile Ad hoc Networks (I MANET) helps to link fixed as well as mobile nodes.

\subsection{MANET challenges}

- The wireless link characteristics are time-varying in nature: There are transmission impediments like fading, path loss, blockage and interference that add to the susceptible behavior of wireless channels. The reliability of wireless transmission is resisted by different factors.

- $\quad$ Limited range of wireless transmission - The limited radio band results in reduced data rates compared to the wireless networks. Hence optimal usage of bandwidth is necessary by keeping low overhead as possible. [9]

Packet losses due to errors in transmission - MANETs experience higher packet loss due to factors such as hidden terminals that results in collisions, wireless channel issues (high bit error rate (BER)), interference, and frequent breakage in paths caused by mobility of nodes, increased collisions due to the presence of hidden terminals and unidirectional links.

- Route changes due to mobility- The dynamic nature of network topology results in frequent path breaks. Frequent network partitions- The random movement of nodes often leads to partition of the network. This mostly affects the intermediate nodes.

\subsection{Routing Protocols}

Routing protocols define a set of rules which governs the journey of message packets from source to destination in a network. In MANET, there are different types of routing protocols each of them is applied according to the network circumstances 


\section{International Journal of Science and Research (IJSR) \\ ISSN (Online): 2319-7064 \\ Index Copernicus Value (2013): 6.14 | Impact Factor (2015): 6.391}

- Proactive Routing Protocols: Proactive routing protocols are also called as table driven routing protocols. In this every node maintain routing table which contains information about the network topology even without requiring it. This feature although useful for datagram traffic, incurs substantial signaling traffic and power consumption. The routing tables are updated periodically whenever the network topology changes. Proactive protocols are not suitable for large networks as they need to maintain node entries for each and every node in the routing table of every node. These protocols maintain different number of routing tables varying from protocol to protocol. There are various well known proactive routing protocols. Example: DSDV, OLSR, WRP etc.

- Reactive Routing Protocols: Reactive routing protocol is also known as on demand routing protocol. In this protocol route is discovered whenever it is needed Nodes initiate route discovery on demand basis. Source node sees its route cache for the available route from source to destination if the route is not available then it initiates route discovery process. The on- demand routing protocols have two major components Ex: DSR, DSDV, AODV. [12]

\subsection{Security Goals}

- Availability: Accessibility implies the benefits are open to approved gatherings at fitting times. Accessibility applies both to information and to administrations. It guarantees the survivability of system administration regardless of refusal of administration assault.

- Confidentiality: It guarantees that computer related resources are gotten to just by approved gatherings. That is, just the individuals who thought to have admittance to something will really get that get to. To keep up secrecy of some private data, we have to keep them mystery from all elements that do not have benefit to get to them. Secrecy is frequently called mystery or protection [6].

- Integrity: Trustworthiness implies that benefits can be altered just by approved gatherings or just in approved way. Change incorporates composing, evolving status, erasing and making. Trustworthiness guarantees that a message being exchanged is never defiled. [5]

- Authentication: Confirmation empowers a hub to guarantee the personality of associate hub it is corresponding with. Validation is basically certification that members in correspondence are confirmed and not impersonators. Validness is guaranteed in light of the fact that just the true blue sender can create a message that will unscramble legitimately with the shared key.

- Authorization: This property relegates diverse access rights to diverse sorts of clients. For instance a system administration can be performed by system overseer just. [4]

\section{Review of Literature}

Watteyne, T. et al [1] "From MANET to IETF ROLL Standardization: A Paradigm Shift in WSN Routing Protocols" In large networks, a data source may not reach the intended sink in a single hop, thereby requiring the traffic to be routed via multiple hops. An optimized choice of such routing path is known to significantly increase the performance of said networks. This holds particularly true for wireless sensor networks (WSNs) consisting of a large amount of miniaturized battery-powered wireless networked sensors required to operate for years with no human intervention. There has hence been a growing interest on understanding and optimizing WSN routing and networking protocols in recent years, where the limited and constrained resources have driven research towards primarily reducing energy consumption, memory requirements and complexity of routing functionalities. To this end, early flooding-based and hierarchical protocols have migrated within the past decade to geographic and self-organizing coordinate-based routing solutions. The former have been brought to standardization through the Internet Engineering Task Force (IETF) Mobile Ad-hoc Networks (MANET) working group; the latter are currently finding their way into standardization through the IETF Routing Over Low power and Lossy networks (ROLL) working group.

Wei Liu et al [2] "DELAR: A Device-Energy-Load Aware Relaying Framework for Heterogeneous Mobile Ad Hoc Networks" This paper addresses energy conservation, a fundamental issue of paramount importance in heterogeneous mobile ad hoc networks (MANETs) consisting of powerful nodes (i.e., P-nodes) as well as normal nodes (i.e., B-nodes). By utilizing the inherent device heterogeneity, we propose a cross-layer designed Device-Energy-Load Aware Relaying framework, named DELAR, to achieve energy conservation from multiple facets, including power-aware routing, transmission scheduling and power control. In particular, we design a novel power-aware routing protocol that nicely incorporates device heterogeneity, nodal residual energy information and nodal load status to save energy.

Jeng, A.A. et al [3] "Adaptive Topology Control for Mobile Ad Hoc Networks" In MANETs, mobile devices are usually powered by batteries with limited energy supplies. Topology control is a promising approach, which conserves energy by either reducing transmission power for each node or preserving energy-efficient routes for the entire network. However, there is empirically a trade-off between the energy efficiency of the nodes and routes in a topology. Besides, it may consume considerable energy to maintain the topology due to node mobility. In this paper, we propose an adaptive topology control protocol for mobile nodes. The protocol allows each node to decide whether to support energyefficient routing or conserve its own energy. Moreover, it can drastically shrink the broadcasting power of beacon messages for mobile nodes. We prove that any reconstruction and change of broadcasting radius converge in four and five beacon intervals, respectively.

Hiranandani, D. et al [4] "MANET protocol simulations considered harmful: the case for benchmarking" In this article, we investigate the current best practices in simulation-based multi-hop wireless ad-hoc network (MANET) protocol evaluation. We extend a prior characterization of the settings and parameters used in MANET simulations by studying the papers published in one of the premier mobile networking conferences between 2006 and 2010. We find that there are still several 


\section{International Journal of Science and Research (IJSR) \\ ISSN (Online): 2319-7064 \\ Index Copernicus Value (2013): 6.14 | Impact Factor (2015): 6.391}

configuration pitfalls which many papers fall victim to, which in turn damages the integrity of the results as well as any research aimed at reproducing and extending these results. We then describe the simulation "design space" of MANET routing in terms of its basic dimensions and corresponding parameters.

Shibo He et al [5] "EMD: Energy-Efficient P2P Message Dissemination in Delay-Tolerant Wireless Sensor and Actor Networks" In this paper, we address the problem of peer-topeer networking for data dissemination among actors in wireless sensor and actor networks (WSANs), which consist of static sensors, responsible for environment monitoring, and mobile actors, in charge of data collection and task performing. This problem has not been received much attention although peer-to-peer networking has achieved great successes in other networks such as the Internet and mobile ad hoc networks (MANETs). Unlike the Internet and MANETs, WSANs contain static sensors that are energyconstrained and actors that cannot communicate with each other directly. These unique characteristics make the data dissemination problem in WSANs extremely challenging. We present an Energy-Efficient Message Dissemination protocol (EMD) to solve this problem in delay-tolerant WSANs. EMD is grounded on a novel principle of "CarryDisseminate-Store-and-Forward" proposed for the first time here. While traveling, a source actor disseminates messages (data) to sensors upon contact, which will store the messages and forward them to other actors when they come into communication range.

\section{Approaches Used}

\subsection{Dynamic Destination-Sequenced Distance}

Vector Routing Protocol (DSDV) DSDV is developed on the basis of Bellman-Ford routing algorithm with some modifications. In this routing protocol, each mobile node in the network keeps a routing table. Each of the routing table contains the list of all available destinations and the number of hops to each. Each table entry is tagged with a sequence number, which is originated by the destination node. Periodic transmissions of updates of the routing tables help maintaining the topology information of the network. If there is any new significant change for the routing information, the updates are transmitted immediately. So the routing information updates might either be periodic or event driven. DSDV protocol requires each mobile node in the network to advertise its own routing table to its current neighbors.

\subsection{Cluster Gateway Switch Routing Protocol (CGSR)}

CGSR uses DSDV protocol as the underlying routing scheme and, hence, it has the same overhead as DSDV. However, it modifies DSDV by using a hierarchical clusterhead-to-gateway routing approach to route traffic from source to destination. Gateway nodes are nodes that are within the communication ranges of two or more cluster heads. A packet sent by a node is first sent to its cluster head, and then the packet is sent from the cluster head to a gateway to another cluster head, and so on until the cluster head of the destination node is reached. The packet is then transmitted to the destination from its own cluster head.

\subsection{Dynamic Source Routing (DSR)}

DSR is a reactive protocol based on the source route approach. In Dynamic Source Routing (DSR), shown in, the protocol is based on the link state algorithm in which source initiates route discovery on demand basis. The sender determines the route from source to destination and it includes the address of intermediate nodes to the route record in the packet. DSR was designed for multi hop networks for small Diameters. It is a beaconless protocol in which no HELLO messages are exchanged between nodes to notify them of their neighbors in the network. [13]

\subsection{Ad Hoc on-Demand Distance Vector Routing (AODV)}

AODV is basically an improvement of DSDV but AODV is a reactive routing protocol instead of proactive. It minimizes the number of broadcasts by creating routes based on demand, which is not the case for DSDV. When any source node wants to send a packet to a destination, it broadcasts a route request (RREQ) packet. The neighboring nodes in turn broadcast the packet to their neighbors and the process continues until the packet reaches the destination. During the process of forwarding the route request, intermediate nodes record the address of the neighbor from which the first copy of the broadcast packet is received. This record is stored in their route tables, which helps for establishing a reverse path.

\section{Conclusion}

MANET is a field of networking that deals with the mobiles nodes of communication. In the MANET position of nodes get dynamically changed in entire iteration. In this procedure various proactive \& reactive protocol are used that provide reliable communication. The major Concern of MANET is energy optimization. The energy consumption problem dynamic clustering or residual energy concept can be used that reduce the energy consumption in MANET. The route discovery mechanism has been used that transmit the RREQ message \& computer then residual energy consumed \& energy available to all nodes for a single route. After calculation of this the best route has been selected that use minimum pops, uses energy and the reactive protocols have been used for transmission of the message by using the best route defined by RDM after transmission of the message the lifetime \& packet delay ratio, packet delay \& throughput of the system has to be analyzed for performs evolutions.

\section{References}

[1] T. Watteyne, A. Molinaro, M. G. Richichi, and M. Dohler, "From MANET to IETF ROLL standardization: A paradigm shift in WSN routing protocols," IEEE Commun. Surv. Tutorials, vol. 13, no. 4, pp. 688-707, 2011.

[2] W. Liu, C. Zhang, G. Yao, and Y. Fang, "DELAR: A device-energy-load aware relaying framework for heterogeneous mobile ad hoc networks," IEEE J. Sel. 


\section{International Journal of Science and Research (IJSR) \\ ISSN (Online): 2319-7064}

Index Copernicus Value (2013): 6.14 | Impact Factor (2015): 6.391

Areas Commun., vol. 29, no. 8, pp. 1572-1584, 2011.

[3] A. A. Jeng and Rong-Hong Jan, "Adaptive Topology Control for Mobile Ad Hoc Networks," IEEE Trans. Parallel Distrib. Syst., vol. 22, no. 12, pp. 1953-1960, 2011.

[4] D. Hiranandani, K. Obraczka, and J. J. Garcia-LunaAceves, "MANET protocol simulations considered harmful: The case for benchmarking," IEEE Wirel. Commun., vol. 20, no. 4, pp. 82-90, 2013.

[5] S. He, X. Li, J. Chen, P. Cheng, Y. Sun, and D. Simplot-Ryl, "EMD: Energy-efficient p2p message dissemination in delay-tolerant wireless sensor and actor networks," IEEE J. Sel. Areas Commun., vol. 31, no. 9, pp. 75-84, 2013.

[6] G. Varaprasad, "New Power-Aware Multicast Algorithm for Mobile Ad Hoc Networks" IEEE Potentials, Issue March, pp. 32-35, 2013.

[7] R. Sanchez-Iborra, M. D. Cano, and J. Garcia-Haro, "Performance evaluation of BATMAN routing protocol for VoIP services: A QoE perspective," IEEE Trans. Wirel. Commun., vol. 13, no. 9, pp. 4947-4958, 2014.

[8] X. Jin, R. Zhang, J. Sun, and Y. Zhang, "TIGHT: A geographic routing protocol for cognitive radio mobile Ad Hoc networks," IEEE Trans. Wirel. Commun., vol. 13, no. 8, pp. 4670-4681, 2014.

[9] G. Jayanthi, "Designing Energy Routing Protocol with Power Consumption Optimization in MANET," IEEE Transactions on Emerging Topics in Computing, vol. 2, no. 2, pp. 192-197, 2014.

[10]Dong-Li Zhang, Wen-cheng Jiao, Zheng Jian-Ling "Research and improvement of Dsr protocol in Ad Hoc Network", Industrial and Information Systems (IIS), vol.1, pp. 242-244, IEEE, 2010.

[11]Zhiyong Shi, Shenquan Zhu, Zhenyu Zhang "Study on application of DSR protocol to mobile communication system", Mobile Technology, Applications and Systems, pp.-5, IEEE, 2005.

[12] Varshney, "Performance improvement of MANET under DSR protocol using swarm optimization", Issues and Challenges in Intelligent Computing Techniques (ICICT), PP. 58-63, IEEE, 2014.

[13] Jhuria, M., Singh, S. "Improve Performance DSR Protocol by Application of Mobile Agent", Communication Systems and Network Technologies (CSNT), pp. 336-340, IEEE, 2014.

[14] Ahmad, S, Awan, I., Waqqas, A.; Ahmad, B. "Performance Analysis of DSR \& Extended DSR Protocols", Modeling \& Simulation, pp. 191-196, IEEE, 2008. 